\begin{abstract}
Yield-per-recruit and spawning-biomass-per-recruit models, are commonly used for evaluating the status of a fishery. In practice, model parameters are themselves usually estimates that are subject to both bias (uncertainty in the mean) and imprecision (uncertainty in the standard deviation). Using Monte Carlo simulation with data for female Japanese eel (Anguilla japonica) from the Kao-Ping River in Taiwan, we examined the sensitivity of such models to different degrees of bias and imprecision in the life history parameters. Positive biases in natural mortality and the von Bertalanffy growth coefficient led to larger relative changes in the mean and standard deviation of estimated fishing-mortality-based biological reference points $\left(F_{\mathrm{BRPs}}\right)$ than did changes under negative biases. Higher degrees of imprecision in parameters did not affect the means of $F_{\mathrm{BRPs}}$, but their standard deviations increased. Composite risks of overfishing depended mainly on the changes in the means of $F_{\mathrm{BRPs}}$ rather than on their standard deviations. Therefore, reducing the biases in key life history parameters, as well as the bias and imprecision in the current rate of fishing mortality, may be the most relevant approach for obtaining correct estimates of the risks of overfishing.
\end{abstract}

Manuscript submitted 22 February 2014. Manuscript accepted 8 May 2015.

Fish. Bull. 113:302-312 (2015).

Online publication date: 2 June 2015. doi: 10.7755/FB.113.3.6

The views and opinions expressed or implied in this article are those of the author (or authors) and do not necessarily reflect the position of the National Marine Fisheries Service, NOAA.

\title{
Sensitivity of yield-per-recruit and spawning- biomass-per-recruit models to bias and imprecision in life history parameters: an example based on life history parameters of Japanese eel (Anguilla japonica)
}

\author{
Yu-Jia Lin' \\ Chi-Lu Sun (contact author) ${ }^{1}$ \\ Yi-Jay Chang 1 \\ Wann-Nian Tzeng ${ }^{2}$ \\ Email for contact author: chilu@ntu.edu.tw \\ 1 Institute of Oceanography \\ National Taiwan University \\ No.1, Sec.4, Roosevelt Road \\ Taipei 10617, Taiwan \\ 2 Department of Environmental Biology and Fisheries Science \\ National Taiwan Ocean University \\ No. 2, Beining Road \\ Keelung 20224, Taiwan
}

Yield-per-recruit (YPR) and spawning-biomass-per-recruit (SPR) models, in which the total yield or spawning biomass of a cohort is standardized for the numbers of recruits, are commonly used in fisheries assessment (Beverton and Holt, 1957; Quinn and Deriso, 1999). They can be used to infer the total yield and spawning biomass of an entire population composed of different cohorts with an assumption of a steady state and with knowledge of equilibrium recruitment (King, 2007). Several fishing-mortality-based biological reference points $\left(F_{\mathrm{BRPs}}\right)$ derived from YPR and SPR models can be used to evaluate whether the yield per recruit is optimal or the spawning biomass per recruit is sufficient for the population to persist under current fishing pressure.

Uncertainties in the parameters of such models are inevitable and result from observation and process error (Charles, 1998). Ignoring uncertainties in parameters can lead to incor- rect estimation of $F_{\mathrm{BRPs}}$, and consequently the examination of fishery status could be misleading, given the cases of the American lobster (Homarus americanus) (Chen and Wilson, 2002), green sea urchin (Strongylocentrotus droebachiensis) (Grabowski and Chen, 2004), Atlantic cod (Gadus morhua) (Jiao et al., 2005), pronghorn spiny lobster (Panulirus penicillatus) (Chang et al., 2009), and Japanese eel (Anguilla japonica) (Lin et al., 2010a).

Estimation of natural mortality $(M)$ is challenging (Vetter, 1988) because both its mean and variance are prone to considerable uncertainty. For example, the estimates of $M$ have varied among different empirical methods (Pascual and Iribarne, 1993; Lin and Sun, 2013). The variances of $M$ estimates also have differed among approaches (e.g., Cubillos et al., 1999, versus Hall et al., 2004; Lin et al., 2012). It is easier to obtain growth information. The von Bertalanffy growth function is an 
often used growth model, but different estimation approaches (e.g., length-frequency methods versus readings of rings in calcified structures; see Lin and Tzeng, 2009, versus Lin and Tzeng, 2010), different regions of study (Helser and Lai, 2004; Keller et al., 2012), and aging errors from annuli readings (Lai and Gunderson, 1987; Cope and Punt, 2007) may lead to biases in the von Bertalanffy growth coefficient $(K)$.

In another example of the potential for uncertainty, biases in the asymptotic length $\left(L_{\infty}\right)$ may result from the regional variation in growth potential in different habitats (Beverton and Holt, 1957), from a latitudinal trend (Helser and Lai, 2004; Keller et al., 2012), or from sampling schemes unrepresentative of the population size structure, because of its high dependency of maximum size in the sample (Formacion et al., 1991; Froese and Binohlan, 2000). In addition, the uncertainty (or multiplicative error) in the growth curve $\left(\varepsilon_{\mathrm{GR}}\right)$ is related to the imprecision in the lengths-at-age scatter at a given age. The current fishing mortality rate $\left(F_{\text {cur }}\right)$ can be estimated from various methods (e.g., Seber, 1982; Quinn and Deriso, 1999; King, 2007) with both the mean and variance of high uncertainty (Chen and Wilson, 2002).

It is essential to incorporate and quantify these uncertainties in the assessment of population dynamics (Hilborn and Walters, 1992; Peterman, 2004). Further, parameter uncertainties can be categorized into bias uncertainty and precision uncertainty. The influence of parameter bias on YPR and SPR models has been investigated since the 1950s (e.g., Beverton and Holt, 1957; Goodyear, 1993; Mace, 1994), but the effects of precision uncertainty have seldom been addressed. The effects of parameter imprecision with a limited number of comparisons (i.e., high or low variation) that were inadequate to reveal a full picture of the sensitivity to bias and imprecision in the parameters have been examined in a few studies (e.g., Restrepo and Fox, 1988; Chen and Wilson, 2002; Chang et al., 2009). Systematic examinations of different degrees of parameter bias and imprecision with a wider range and finer resolution could provide detailed information about their effects on model outputs (e.g., Goodyear, 1993), but few such examinations exist for per-recruit models.

It is difficult to elucidate the sensitivity of perrecruit models to parameter bias and imprecision through analysis of the formulae because the effects of the parameters on $F_{\mathrm{BRPs}}$ are nonlinear with complicated forms (Schnute and Richard, 1998). Alternatively, a sensitivity analysis through the use of a Monte Carlo simulation can be used to examine their effects on the estimation of $F_{\mathrm{BRPs}}$, on fishery status, and on management implications (Jiao et al., 2005). Therefore, the objective of this study was to evaluate the effects of different degrees of bias, represented by the differences in parameter means, and of imprecision, represented by the differences in parameter standard deviations (SDs), in the YPR and SPR model results, specifically in the $F_{\mathrm{BRPs}}$ and the resultant composite risks of overfishing.

\section{Materials and methods}

To better understand the sensitivity of outputs from the YPR and SPR models, we changed the mean or SD of selected parameters one at a time over large ranges with fine increments (from 5\% to $95 \%$ with increments of $5 \%$ for cases of under-specification and from $150 \%$ to $1000 \%$ with increments of $50 \%$ for cases of overspecification). The following parameters were selected: 1) $M$;2) von Bertalanffy growth coefficients of $K$ and $L_{\infty}$; and 3) $F_{\text {cur. }}$. In addition, the multiplicative error in the growth curve $\left(\varepsilon_{\mathrm{GR}}\right)$ and length-weight relationship $\left(\varepsilon_{\mathrm{LW}}\right)$ were selected as well. In this study, we used information on the mean and SD values of these parameters from previous studies conducted during 1998-2006 on the life history and fishery of the Japanese eel in the Kao-Ping River in Taiwan (Lin and Tzeng 2009; Lin et al. 2010a, 2010b) to examine the sensitivity of model results to effects of parameter bias and imprecision with a Monte Carlo simulation.

We used wide ranges for the parameters for 3 reasons: 1) the scale of boundaries used; a factor of 10 was applied in a classical study (figure 4 in Goodyear, 1993); 2 ) a wide range is more general because it can include all likely ranges; and 3) in our previous study (Lin and Sun, 2013), we found that the variation in estimates of $M$ from several different indirect methods can vary from 0.10 year to 2.13 year. Also, the difference in $K$ can be large, especially when different approaches are involved. For the same region, for example, in Kao-Ping River, $K$ has varied from 0.12 /year to 0.38 /year, depending on whether methods based on otoliths or length frequencies were applied (Lin and Tzeng, 2009, 2010).

\section{YPR and SPR models}

The YPR and SPR models were calculated according to the formulae in Quinn and Deriso (1999):

$$
\begin{gathered}
Y P R=e^{-M\left(t_{c}-t_{r}\right)} \int_{t_{c}}^{t_{\max }} F(t) N(t) W(t) d t \text { and } \\
S P R=\int_{t_{c}}^{t_{\max }} N(t) W(t) S(t) d t,
\end{gathered}
$$

where $F(t)=$ the fishing mortality at age $t$;

$N(t)=$ population size in numbers at age $t$;

$W(t)=$ the weight at age $t$;

$S(t)=$ the maturation proportion at age $t$;

$t_{\mathrm{r}}=$ the age at recruitment;

$t_{\mathrm{c}}=$ the age at first capture; and

$t_{\text {max }}=$ the maximum age (the formulae for $F(t)$, $N(t), W(t)$, and $S(t)$ are shown in Table 1$)$.

Because, in Kao-Ping River, females were 4 times more abundant than males (Han and Tzeng, 2006) and because females contribute more directly to spawning biomass, the parameters of female Japanese eels were used in this study; they were also used in Lin et al. (2010a). Estimates of means and corresponding SDs of the parameters were derived from our previous works (Lin and Tzeng, 2009; Lin et al.,2010b). 


\section{Table 1}

Formulae, estimates of model parameters, and corresponding simulated random errors for the length-weight relationship ( $a$ and $b$ are coefficients, and $\varepsilon_{L W}=$ the corresponding multiplicative error), $\mathrm{N}=$ the population size in numbers; von Bertalanffy growth function ( $K=$ the growth coefficient (/year), $L_{\infty}=$ the asymptotic length $(\mathrm{mm}), t_{0}=$ the theoretical age at length of zero (year), and $\varepsilon_{\mathrm{GR}}=$ the multiplicative error of the growth curve), $M=$ the natural mortality (/year); $\varepsilon_{\mathrm{M}}=$ the standard deviation of $M ; F_{\text {cur }}=$ current fishing mortality (/year); fishing process under gear selection ( $\alpha_{0}$ and $\alpha_{1}=$ selection curve coefficients), and maturation process of female Japanese eel (Anguilla japonica) in the lower reach of the Kao-Ping River in southern Taiwan $\left(\beta_{0}\right.$ and $\beta_{1}=$ the maturation curve coefficients, and $\varepsilon_{\beta 0}$ and $\varepsilon_{\beta 1}$ =the standard error for $\beta_{0}$ and $\beta_{1}$, respectively). The means and standard deviations of the parameters are from previous studies conducted in 1998-2006 on life history parameters and the fishery of Japanese eel in the KaoPing River, Taiwan (Lin and Tzeng 2009, Lin et al. 2010a, 2010b).

\begin{tabular}{|c|c|}
\hline Formula & Parameter \\
\hline \multicolumn{2}{|l|}{ Weight at age $t, W(t)$} \\
\hline$W(t)=a L(t)^{b} e^{\varepsilon_{L W}}$ & $\begin{array}{l}a, b=4.56 \times 10^{-8}, 3.55 \\
\varepsilon_{\mathrm{LW}} \sim \mathrm{N}\left(0,3.93 \times 10^{-4}\right)\end{array}$ \\
\hline$L(t)=L_{\infty}\left[1-e^{-K\left(t-t_{0}\right)}\right] e^{\varepsilon_{G R}}$ & $\begin{array}{l}L_{\infty}, K, t_{0}=1024,0.12,-0.69 \\
\varepsilon_{\mathrm{GR}} \sim \mathrm{N}\left(0,3.10 \times 10^{-2}\right)\end{array}$ \\
\hline \multicolumn{2}{|l|}{ Population size in number at age $t, N(t)$} \\
\hline$N(t)=e^{-\left[F(t)+\left(M+\varepsilon_{M}\right)\right]\left(t-t_{r}\right)}$ & $\begin{array}{l}M=0.18 / \text { year } \\
\varepsilon_{\mathrm{M}} \sim \mathrm{N}\left(0,1.98 \times 10^{-5}\right)\end{array}$ \\
\hline \multicolumn{2}{|c|}{ Fishing process under logistic gear selection, $F(t)$} \\
\hline$F(t)=F_{c u r} \times \frac{e\left[\alpha_{0}+\alpha_{1} L(t)\right]}{1+e\left[\alpha_{0}+\alpha_{1} L(t)\right]}$ & $\begin{array}{l}F_{\text {cur }} \sim \text { Gamma } \text { with mean of } \\
0.12 \text { and variance of } \\
1.06 \times 10^{-2}, \alpha_{0}, \alpha_{1}=-4,0.02\end{array}$ \\
\hline \multicolumn{2}{|c|}{ Maturation (silvering) proportion at age $t, S(t)$} \\
\hline$S(t)=\frac{e^{\left[\left(\beta_{0}+\varepsilon_{\beta_{0}}\right)+\left(\beta_{1}+\varepsilon_{\beta_{1}}\right) L(t)\right]}}{1+e^{\left[\left(\beta_{0}+\varepsilon_{\beta_{0}}\right)+\left(\beta_{1}+\varepsilon_{\beta_{1}}\right) L(t)\right]}}$ & $\begin{array}{l}\beta_{0}, \beta_{1}=-13.31,0.02 \\
\varepsilon_{\beta_{0}} \sim \mathrm{N}(0,1.36) \text { and } \\
\varepsilon_{\beta_{1}} \sim \mathrm{N}\left(0,4.04 \times 10^{-6}\right)\end{array}$ \\
\hline \multicolumn{2}{|l|}{ Others } \\
\hline Maximum observed age, $t_{\max }$ & 16 years \\
\hline Length and age at recruitment, $L_{\mathrm{r}}, t_{\mathrm{r}}$ & $55 \mathrm{~mm}, 0.489$ year \\
\hline Length and age at first capture, $L_{\mathrm{c}}, t_{\mathrm{c}}$ & $200 \mathrm{~mm}, 1.15$ year \\
\hline
\end{tabular}

Four $F_{\text {BRPs }}$ were calculated to compare with $F_{\text {cur }}\left(0.120 /\right.$ year, Lin et al., 2010b): $F_{\max }$, the fishing mortality at which yield per recruit is at its maximum; $F_{0.1}$, the fishing mortality at which the increase of yield per recruit is only $10 \%$ of the increase of yield per recruit when fishing mortality is zero (King, 2007); and $F_{30 \%}$ and $F_{50 \%}$, the fishing mortality rates at which the SPR is $30 \%$ and $50 \%$ of the SPR when fishing mortality is zero (Goodyear, 1993).

The reference points $F_{\max }$ and $F_{0.1}$ can be regarded as boundaries in order to constrain harvesting below the level within which the fish population can produce maximum sustainable yield (United Nations, 1995), and they usually are used as limiting and precautionary indicators of growth overfishing, respectively; fishing mortality rates above $F_{\max }$ indicate that fish are caught before they reach optimal size given the rate of natural mortality and their growth rate (Gabriel and Mace, 1999; Quinn and Deriso, 1999). Fishing mortalities at $30 \%$ and $50 \%$ of SPR, compared with SPR at $F=0$, are considered to be the limiting and precaution- ary levels for anguillid eels (ICES $\left.{ }^{1}\right)$. Therefore, $F_{30 \%}$ and $F_{50 \%}$ were considered the limiting and precautionary reference points for recruitment overfishing, where the spawners are insufficient in numbers to produce enough offspring to replace themselves (Sissenwine and Shepherd, 1987).

\section{Incorporation of uncertainties in parameters}

We used Monte Carlo simulation, which has been widely applied to other species (e.g., Chen and Wilson, 2002; Grabowski and Chen, 2004; Chang et al., 2009; Lin et al., 2010a) to incorporate the uncertainties of the parameters into the models. In each run, random errors for natural mortality $\left(\varepsilon_{\mathrm{M}}\right)$ and for coefficients in the logistic maturation curve $\left(\varepsilon_{\beta_{0}}\right.$ and $\left.\varepsilon_{\beta_{1}}\right)$, as well as $\varepsilon_{\mathrm{LW}}$

${ }^{1}$ ICES (International Council for the Exploration of the Sea). 2002. Report of the ICES/EIFAC Working Group on Eels. ICES Council Meeting (C.M.) Documents 2002/ACFM:03, 55 p. [Available at website.] 


\section{Table 2}

Summary of the 9 scenarios for evaluation of the sensitivity of the results from models of yield per recruit and spawning biomass per recruit to different degrees of bias and imprecision in several parameters: natural mortality $(M)$, von Bertalanffy growth coefficient $(K)$, asymptotic length $\left(L_{\infty}\right)$, and current fishing mortality rate $\left(F_{\text {cur }}\right)$. Also the multiplicative error in the growth curve $\left(\varepsilon_{\mathrm{GR}}\right)$ and the length-weight (LW) relationship $\left(\varepsilon_{\mathrm{LW}}\right)$ are modeled. In each scenario, the mean or standard deviation (SD) of only one parameter was changed.

\begin{tabular}{llll}
\hline Scenario & Description & Parameter & Range \\
\hline 1 & Standard scenario & Unchanged & $100 \%$ \\
2 & Mean of $M$ & $M$ & $10-1000 \%$ \\
3 & SD of $M$ & $\varepsilon_{\mathrm{M}}$ & $10-1000 \%$ \\
4 & Mean of $K$ & $K$ & $10-1000 \%$ \\
5 & Mean of $L_{\infty}$ & $L_{\infty}$ & $50-200 \%$ \\
6 & Error in growth curve & $\varepsilon_{\mathrm{GR}}$ & $10-1000 \%$ \\
7 & Error in LW relationship & $\varepsilon_{\mathrm{LW}}$ & $10-1000 \%$ \\
8 & Mean of $F_{\text {cur }}$ & $F_{\text {cur }}$ & $10-1000 \%$ \\
9 & SD of $F_{\text {cur }}$ & $\varepsilon_{\mathrm{F}_{\text {cur }}}$ & $10-1000 \%$ \\
& & & \\
\hline
\end{tabular}

and $\varepsilon_{\mathrm{GR}}$, were generated from normal distribution with the means and variances listed in Table 1 . These random errors were incorporated into the YPR and SPR formulae provided in Table 1 , and then $4 F_{\mathrm{BRPs}}\left(F_{\max }\right.$, $F_{0.1}, F_{30 \%}$, and $\left.F_{50 \%}\right)$ were calculated. For each simulation scenario, this process was repeated 5000 times to produce 5000 corresponding sets of $F_{\mathrm{BRP}}$ values from which the empirical distributions of the $4 F_{\mathrm{BRPs}}$ were generated and composite risks were calculated.

\section{Calculation of composite risks}

Because $F_{\text {cur }}$ and the $F_{\mathrm{BRPs}}$ are not fixed constants, we applied composite risk analysis that allowed for the incorporation of the uncertainty in both indicator and management reference points (Prager et al., 2003; Jiao et al., 2005). By the discrete approach proposed by Jiao et al. (2005), the composite risks were calculated as the expected probability of one random variable being larger than another. Let $f(x)$ be the empirical probability density function of the $F_{\mathrm{BRP}}$ of interest (e.g., $F_{\max }$ ) and $g(y)$ be the empirical probability density function of $F_{\text {cur }}$ with a corresponding cumulative density function of $G(y)$ and then, let $\Delta x$ be a small increase in $x$ (i.e., the $F_{\mathrm{BRP}}$ ), and by summing $x$ over its range, the composite risk of $F_{\text {cur }}$ exceeding $F_{\text {BRP }}$ is calculated with the following equation:

$$
P\left(F_{\text {cur }}>F_{B R P}=1-\sum_{-\infty}^{\infty}[G(x) f(x)] \Delta x\right.
$$

Here, we assume a gamma distribution for $F_{\text {cur }}$ because 1) our previous study (Lin and Tzeng, 2008) indicated that gamma distribution fitted better for the distribution of fishing efforts, 2) it produces nonnegative values, and 3) it is flexible in shape. For details in cal- culation of composite risks in discrete approach, refer to Jiao et al. (2005).

\section{Sensitivity analysis}

For the sensitivity analysis, the parameter values in Table 1 were set as the standard scenario (scenario 1; Table 2). The parameters for which mean and $S D$ values may potentially affect results of YPR and SPR models were investigated in 9 scenarios, in which the mean or SD of only one parameter was changed (Table 2): the mean of $M$ (scenario 2) and its $\mathrm{SD}\left(\varepsilon_{\mathrm{M}}\right.$, scenario 3 ), the mean of $K$ (scenario 4 ), and the mean of $L_{\infty}$ (scenario 5 ). In practice, the bias in $K$ can result from aging error and the use of different estimation approaches, and the bias in $L_{\infty}$ often results from unrepresentative sampling schemes. Therefore, we assumed that the sources of biases in $K$ and $L_{\infty}$ are unrelatedand they were modeled independently. Because modeling the estimation errors in the coefficients $K$ and $L_{\infty}$ can lead to underestimation of the actual uncertainty in the data (Lin et al., $2012)$, the error was modeled on the growth curve $\left(\varepsilon_{\mathrm{GR}}\right)$ rather than coefficients (scenario 6). The remaining scenarios are $\varepsilon_{\mathrm{LW}}$ (scenario 7) and the mean and SD values of $F_{\text {cur }}$ and $\varepsilon_{\text {Fcur }}$ (scenarios 8 and 9 ).

For scenarios 1-7, the mean and SD values of the parameters, except for $L_{\infty}$, were decreased to $10 \%$ with an increment of $5 \%$ or were increased to $1000 \%$ with an increment of $50 \%$ to cover the possible magnitudes of biological variation (as applied in Goodyear, 1993). Because information about $L_{\infty}$ can be obtained from the maximum observed length in the data, that parameter may be subject to less bias and imprecision and was set from $50 \%$ to $100 \%$ with an increment of $5 \%$ and from $100 \%$ to $200 \%$ with an increment of $10 \%$ (Table 2). For scenarios 8 and 9, because of less computational load (calculation of an $F_{\mathrm{BRP}}$ is independent of $F_{\text {cur }}$, and, therefore, we needed to compute only composite risks of overfishing), we used finer increments: the mean and SD of $F_{\text {cur }}$ were decreased to $10 \%$ with an increment of $1 \%$ or increased to $1000 \%$ with an increment of $10 \%$.

The relative change ( $\mathrm{RC}$ ) between the mean or SD of one $F_{\mathrm{BRP}}$ for increment $i$ in scenario $j\left(R C_{F_{B R P}}^{i j}\right)$ and the mean or SD of that $F_{\mathrm{BRP}}$ in the standard scenario was used to quantify the sensitivity of a given $F_{\mathrm{BRP}}$ for all scenarios except 8 and 9 . RC is defined with the following equation:

$$
R C_{F_{B R P}}^{i j}=100 \times T_{F_{B R P}}^{i j} \times\left(T_{F_{B R P}}^{S}\right)^{-1}
$$

Where $T_{F_{B R P}}^{i j}=$ the statistic (mean or SD) of the $F_{\mathrm{BRP}}$ of interest (e.g., $F_{\max }$ ) for increment $i$ in scenario $j$; and 


\section{Table 3}

Mean and standard deviation (SD) per year of 4 fishery-mortality-based reference points $\left(F_{\mathrm{BRPs}}\right)$ - the fishing mortality at which yield per recruit is at its maximum $\left(F_{\max }\right)$, the fishing mortality at which the increase of yield per recruit is only $10 \%$ of the increase of yield per recruit when fishing mortality is zero $\left(F_{0.1}\right)$, and the fishing mortality rates at which the spawning biomass per recruit (SPR) is $30 \%$ and $50 \%$ of the SPR when fishing mortality is zero $\left(F_{30 \%}\right.$ and $\left.F_{50 \%}\right)$-and the composite risks, $P\left(F_{\text {cur }}>F_{\mathrm{BRP}}\right)$, calculated as percentages, of current fishing mortality, $F_{\text {cur }}\left(0.120 /\right.$ year), exceeding $F_{\mathrm{BRPs}}$ after repeating the standard scenario 1000 times. Numbers in the parentheses provide the ranges of relative changes in means and SDs from random Monte Carlo simulation with data for female Japanese eel (Anguilla japonica) in the Kao-Ping River in southern Taiwan.

\begin{tabular}{lcc}
\hline$F_{\text {BRP }}$ & Mean & SD \\
\hline$F_{\max }$ & $0.156(99.95-100.04 \%)$ & $1.6 \times 10^{-3}(97.23-103.89 \%)$ \\
$F_{0.1}$ & $0.111(99.96-100.03 \%)$ & $9.2 \times 10^{-4}(97.24-103.90 \%)$ \\
$F_{30 \%}$ & $0.129(99.97-100.03 \%)$ & $8.7 \times 10^{-4}(97.32-104.44 \%)$ \\
$F_{50 \%}$ & $0.073(99.97-100.03 \%)$ & $4.6 \times 10^{-4}(97.31-104.43 \%)$ \\
& & \\
$P\left(F_{\text {cur }}>F_{\max }\right)$ & 13.44 & 0.47 \\
$P\left(F_{\text {cur }}>F_{0.1}\right)$ & 56.44 & 0.70 \\
$P\left(F_{\text {cur }}>F_{30 \%}\right)$ & 35.48 & 0.65 \\
$P\left(F_{\text {cur }}>F_{50 \%}\right)$ & 94.31 & 0.32 \\
& &
\end{tabular}

$$
\begin{aligned}
T_{F_{B R P}}^{S}= & \text { the same statistic of the same } F_{\mathrm{BRP}} \text { from } \\
& \text { the standard scenario. }
\end{aligned}
$$

The sensitivity of an $F_{\mathrm{BRP}}$ also has to be distinguished from the random variation that results from Monte Carlo simulation, namely the variation that results from the incorporation of random errors in $M$, lengthweight relationship, and maturation process. To understand the scale of this random variation, the standard scenario (scenario 1, without any biasesor imprecision in parameters) was repeated 1000 times, producing1000 corresponding RC values for the means and SDs of $F_{\text {BRPs. }}$. The minimum and maximum values of $\mathrm{RC}$ from these 1000 repetitions of scenario 1 were used as the lower and upper limits of random variation from the Monte Carlo simulation. The effect of changes in the mean or SD of a parameter was considered significant only when its RC is lower than the minimum or larger than the maximum RC. All the computations and simulations were completed in $R$, vers. 3.1.0 ( $R$ Core Team, 2014).

\section{Results}

\section{Magnitude of the random variation from Monte Carlo simulation}

Running the Monte Carlo simulation of the standard scenario 1000 times produced RC values for the means of $F_{\max }, F_{0.1}, F_{30 \%}$, and $F_{50 \%}$ that ranged from $99.95 \%$ to $100.04 \%$, from $99.96 \%$ to 100.03 , from $99.97 \%$ to 100.03 , and from $99.99 \%$ to $100.03 \%$, respectively (Table 3 ). The SD values of the $F_{\text {BRPs }}$ had larger $\mathrm{RC}$ values that ranged from $97.23 \%$ to $103.89 \%$ for $F_{\max }$, from $97.24 \%$ to $103.90 \%$ for $F_{0.1}$, from $97.32 \%$ to $104.44 \%$ for $F_{30 \%}$, and from $97.31 \%$ to $104.43 \%$ for $F_{50 \%}$ (Table $3)$. Therefore, variation of $\mathrm{RC}$ within $0.05 \%$ for the mean and within $4.50 \%$ for the SD of the $F_{\mathrm{BRP}}$ was applied as the criterion for significance of effects; that is, we considered the effect of changing one parameter on the $F_{\mathrm{BRP}}$ to be significant when the resultant $\mathrm{RC}$ was wider than this criterion.

\section{Sensitivity of reference points from the YPR model}

The means of $F_{\text {BRPs }}$ from the YPR model, namely $F_{\max }$ and $F_{0.1}$, were sensitive to both the means of $M$ and $K$, which had similar trends but different magnitudes (Fig. 1, left panels). The RC values for the means of $F_{\max }$ and $F_{0.1}$ decreased slowly to $75-80 \%$ when $M$ and $K$ decreased to $5 \%$. As $M$ and $K$ increased to $1000 \%$, their $\mathrm{RC}$ values rose with an accelerating trend, especially for $F_{\max }$, to more than $12,000 \%$ and $1100 \%$, respectively. However, the means of $F_{\max }$ and $F_{0.1}$ were not sensitive to changes in $L_{\infty}$ and $\varepsilon_{\mathrm{GR}}$ because their RC varied less than $0.05 \%$. The $\varepsilon_{\mathrm{M}}$ had marginal effects on the mean of $F_{\text {max }}$ and $F_{0.1}$, given that the RC was around $100.6 \%$ when $\varepsilon_{\mathrm{M}}$ became $1000 \%$ (Fig.1, left panels). The SD values of $F_{\max }$ and $F_{0.1}$ were also sensitive to the means of $M$ and $K$ with similar accelerating trends. The SD of $F_{\max }$ was more sensitive to changes in $M$ and $K$ than was the SD of $F_{0.1}$ (Fig. 1 , right panels). In addition, the SD values of $F_{\max }$ and $F_{0.1}$ were nearly related linearly to $\varepsilon_{M}$. Similar to the means, the SD values of $F_{\text {max }}$ and $F_{0.1}$ did not show significant sensitivity to $L_{\infty}$ and $\varepsilon_{\mathrm{GR}}$, and their RC values did not exceed the significance level of $4.50 \%$.

\section{Sensitivity of reference points from the SPR model}

The means of $F_{\text {BRPs }}$ from the SPR model, $F_{30 \%}$, and $F_{50 \%}$, were sensitive to the mean of $M$, but their RC values (from $90 \%$ to $170 \%$; Fig. 2, left panels) were smaller than those of $F_{\max }$ and $F_{0.1}$. When $K$ decreased to $40 \%$, the RC for the mean of $F_{30 \%}$ and $F_{50 \%}$ reached a minimum of around $80 \%$. As $K$ increased to $1000 \%$, the RC of the means of $F_{30 \%}$ and $F_{50 \%}$ increased to $300 \%$ and $400 \%$. They also were affected by $L_{\infty}$ such that the RC was around $85-125 \%$ as $L_{\infty}$ increased from $50 \%$ to $200 \%$. Neither $\varepsilon_{\mathrm{M}}$ nor $\varepsilon_{\mathrm{GR}}$ affected the means of $F_{30 \%}$ and $F_{50 \%}$. The SD values of $F_{30 \%}$ and $F_{50 \%}$ showed the highest sensitivity to the mean of $M$, with 


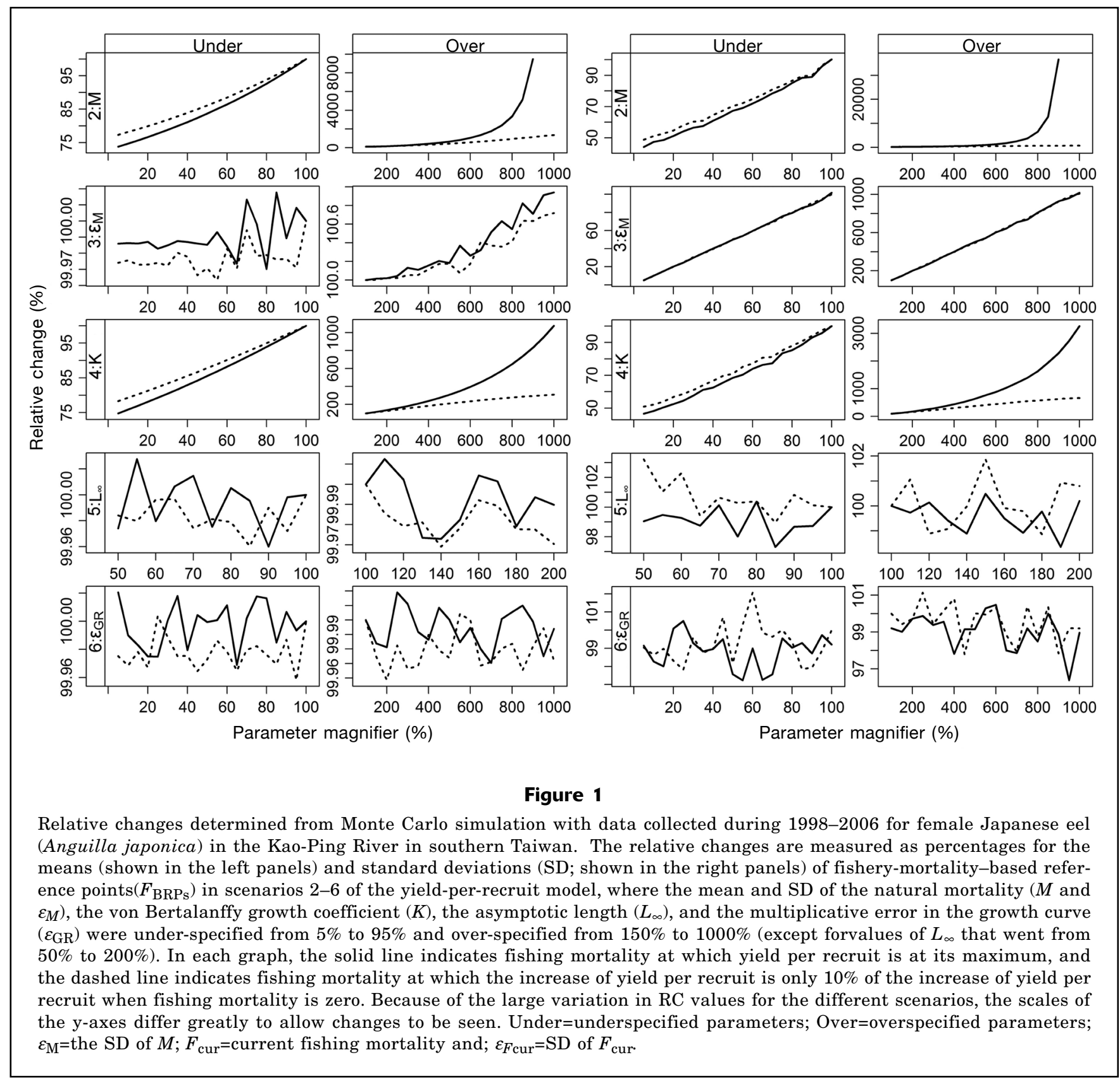

the RC changing from $70 \%$ to around $4000 \%$. The parameter $K$ had high influence on the SD values of $F_{30 \%}$ and $F_{50 \%}$, with RC changing from $20 \%$ to $1500 \%$ (Fig. 2 , right panels). The parameters $\varepsilon_{\mathrm{M}}$ and $L_{\infty}$ had considerable effects on the SD values of $F_{30 \%}$ and $F_{50 \%}$, with RC ranging from $90 \%$ to $400 \%$ and from $40 \%$ to $150 \%$, respectively. The SDs of $F_{30 \%}$ and $F_{50 \%}$ were not affected by $\varepsilon_{\mathrm{GR}}$.

\section{Sensitivity of the composite risks of overfishing}

The composite risks of growth overfishing $\left(F_{\text {cur }}\right.$ exceeding $F_{\max }$ and $F_{0.1}$ ) decreased with increasing means of $M$ and $K$ and did not depend on changes in $L_{\infty}, \varepsilon_{\mathrm{GR}}$, and $\varepsilon_{\mathrm{M}}$ (Fig. 3). The risks of recruitment overfishing ( $F_{\text {cur }}$ exceeding $F_{30 \%}$ and $F_{50 \%}$ ) showed the greatest sensitivity to $K$ followed by $M$. The risk of recruitment overfishing was affected also by changes in $L_{\infty}$ but with less sensitivity. The parameters $\varepsilon_{\mathrm{M}}$ and $\varepsilon_{\mathrm{GR}}$ did not contribute to noticeable changes in the risks of overfishing. The risks of both growth and recruitment overfishing showed strong sensitivity to both the mean and SD of $F_{\text {cur. }}$ As the mean of $F_{\text {cur }}$ increased from around $150 \%$ to $225 \%$, the risks approached $100 \%$. The risks of overfishing decreased with increasing SD for $F_{\text {cur. }}$ 

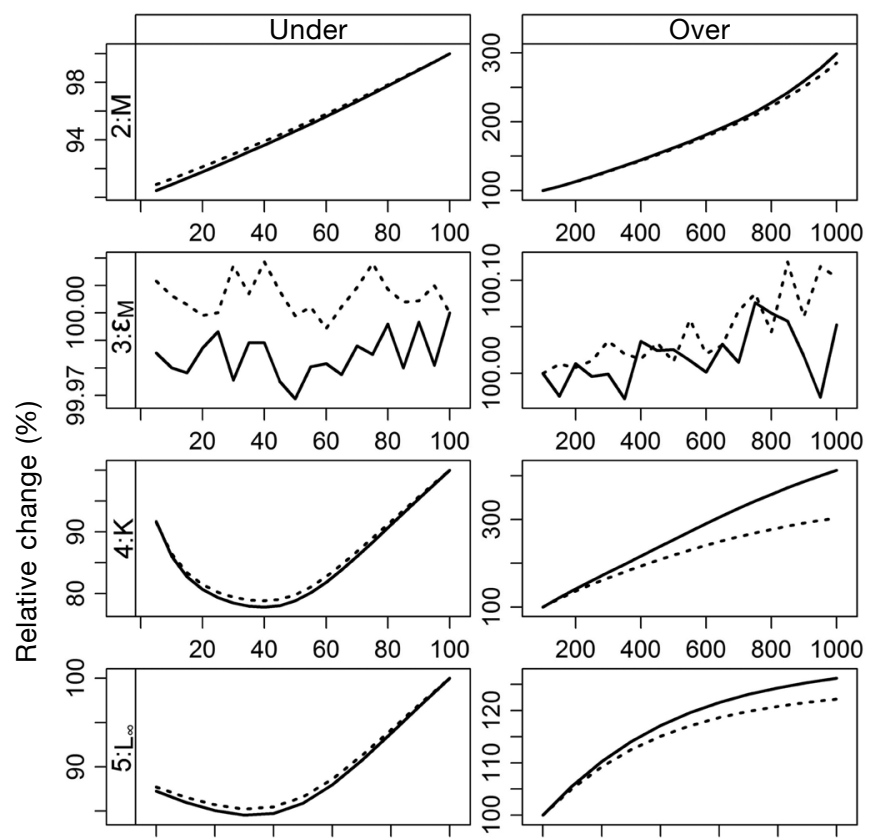

$\begin{array}{llllll}50 & 60 & 70 & 80 & 90 & 100\end{array}$
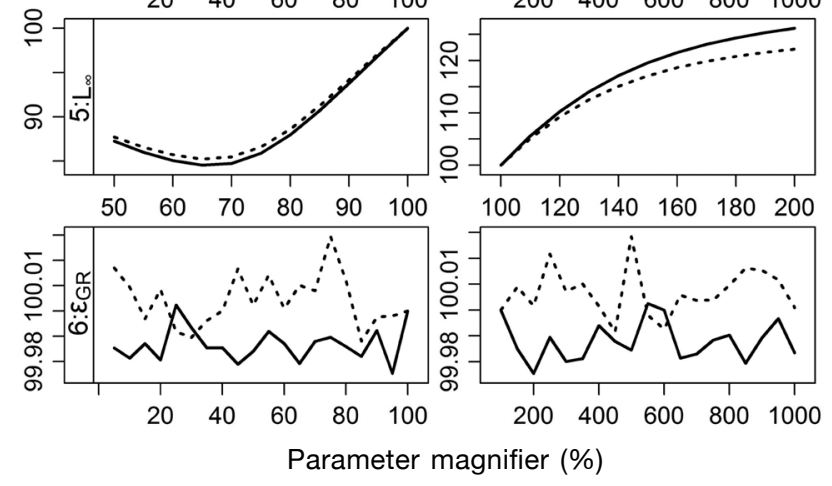
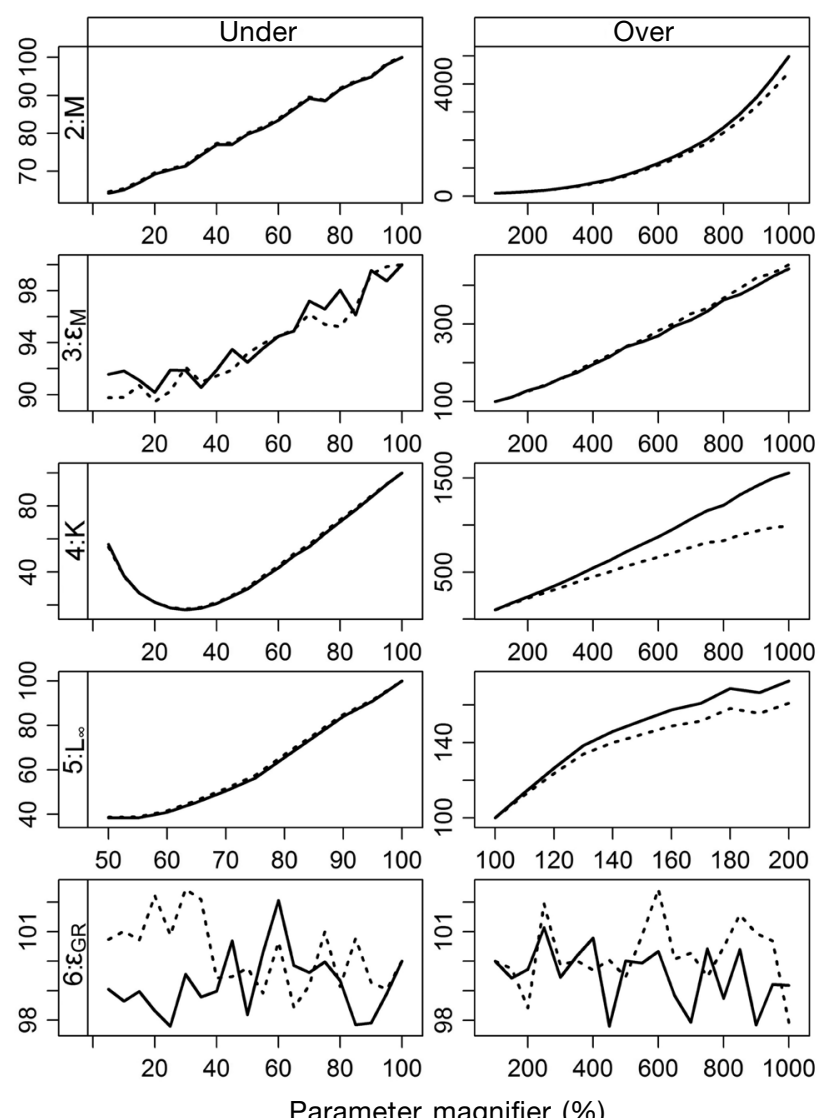

Figure 2

Relative changes from the Monte Carlo simulation with data collected during 1998-2006 for female Japanese eel (Anguilla japonica) in the Kao-Ping River in southern Taiwan. The relative changes are measured as percentages, for the means (shown in the left panels) and SDs (shown in the right panels) of fishery-mortality-based reference points $\left(F_{\mathrm{BRPs}}\right)$ in scenarios 2-6 of the spawning-biomass-per-recruit model, where the mean and SD of the natural mortality $\left(M\right.$ and $\left.\varepsilon_{\mathrm{M}}\right)$, the von Bertalanffy growth coefficient $(K)$, the asymptotic length $\left(L_{\infty}\right)$, and the multiplicative error in the growth curve ( GR) were under-specified from $5 \%$ to $95 \%$ and over-specified from $150 \%$ to $1000 \%$ (except forvalues of $L_{\infty}$ that went from $50 \%$ to $200 \%)$. In each graph, the solid line indicates the fishing mortality rate at which the spawning biomass per recruit (SPR) is $30 \%$ of the SPR when fishing mortality is zero, and the dashed line indicates the fishing mortality rate at which the SPR is $50 \%$ of the SPR when fishing mortality is zero. Because of the large variation in RC values for the different scenarios, the scales of the $y$-axes differ greatly to allow changes to be seen. Under=underspecified parameters; Over=overspecified parameters.

\section{Effects of multiplicative error in the length-weight relationship}

The changes in $\varepsilon_{\mathrm{LW}}$ resulted in nonsignificant changes in the mean and SD of the $4 F_{\text {BRPs. }}$. They also did not affect the composite risks of exceeding these $F_{\text {BRPs }}$.

\section{Discussion}

\section{Effects of uncertainty in mean and SD of natural mortality}

Estimates of the mean of $M$ are usually highly uncertain, possibly because of the lack of appropriate data for direct estimation, data such as those from tag-recapture experiments (Vetter, 1988). As $M$ increases, the YPR curve becomes flatter with a decreasing maximum value and decreasing slope at the origin (fig. 17.18 in Beverton and Holt, 1957). This change in the shape of YPR curve accounts for the nonlinear accelerating trend of $F_{\max }$ and $F_{0.1}$ found in our study. Uncertainty in the mean (bias) of $M$ can also cause significant biases in results of the model for SPR, particularly at a high level of fishing mortality (Goodyear, 1993). A higher mean of $M$ led to a steeper SPR curve, but the general shape of the curve was not altered, explaining the lower sensitivity of $F_{30 \%}$ and $F_{50 \%}$ to $M$. On the other hand, the SD (imprecision) of $M$ resulted 
in significant changes only in the SDs of the 4 $F_{\text {BRPs }}$ examined. Therefore, as indicated in the Appendix Figure, the risks of both growth and recruitment overfishing were affected more by the changes in the mean of $M$ than by changes in the SD. The bias in $M$ seems to be more important than the imprecision in $M$ in influencing the risks of overfishing.

\section{Effects of uncertainty in growth parameters}

The shape of the YPR curve also was altered by $K$ (fig. 17.22 in Beverton and Holt, 1957). The flattening of the YPR curve with decreasing $K$ accounts for the accelerating increase in the mean and SD of $F_{\max }$. On the other hand, Beverton and Holt (1957) found that the changes in $W_{\infty}$ (equivalent to $L_{\infty}$ under the same lengthweight relationship) did not affect the shape of the YPR curve. This insensitivity of the YPR shape on $L_{\infty}$ explains our finding that changes in $L_{\infty}$ did not affect the mean and SD of $F_{\text {max }}$ and $F_{0.1}$ or the corresponding risk of growth overfishing.

The peaks in the values of $\mathrm{RC}$ in the means and SDs of $F_{30 \%}$ and $F_{50 \%}$ when $K$ and $L_{\infty}$ were around $40-60 \%$ possibly resulted from the use of a length-dependent maturation curve for anguillids (Davey and Jellyman, 2003) in the calculation of SPR. In the cases with extremely small values of $K$ or $L_{\infty}$, the proportion of mature eels was close to zero even at the maximum age (16 years). A very small part of the spawning biomass was lost because of fishing, consequently resulting in higher $F_{30 \%}$ and $F_{50 \%}$ values.

\section{Effects of current fishing mortality}

The bias and imprecision in $F_{\text {cur }}$ played an important role in determining risks of overfishing. Greater effects due to changes in the mean of $F_{\text {cur }}$ on the risks of both growth and recruitment overfishing were expected because differences in the mean played an important part in influencing the composite risk, as in the example of 2 standard normal random variables (Appdx Fig.). Given the same difference in the means, a larger SD leads to lower composite risks (Appendix. Figure, panels B and D), accounting for the observed decreasing risks of overfishing with increasing $\varepsilon_{\text {Fcur. }}$. Given that $F_{\text {cur }}$ is from the gamma distribution, decreasing composite risks with increasing $\varepsilon_{\text {Fcur }}$ also resulted in the convergence of 4 risks of overfishing. This finding indicates that a high $\varepsilon_{\text {Fcur }}$ value may mask the distinction between target (usually $F_{0.1}$ or $\left.F_{50 \%}\right)$ and threshold $\left(F_{\max }\right.$ or $\left.F_{30 \%}\right) F_{\text {BRPs }}$ because the risks of target and threshold $F_{\text {brp }}$ are similar.

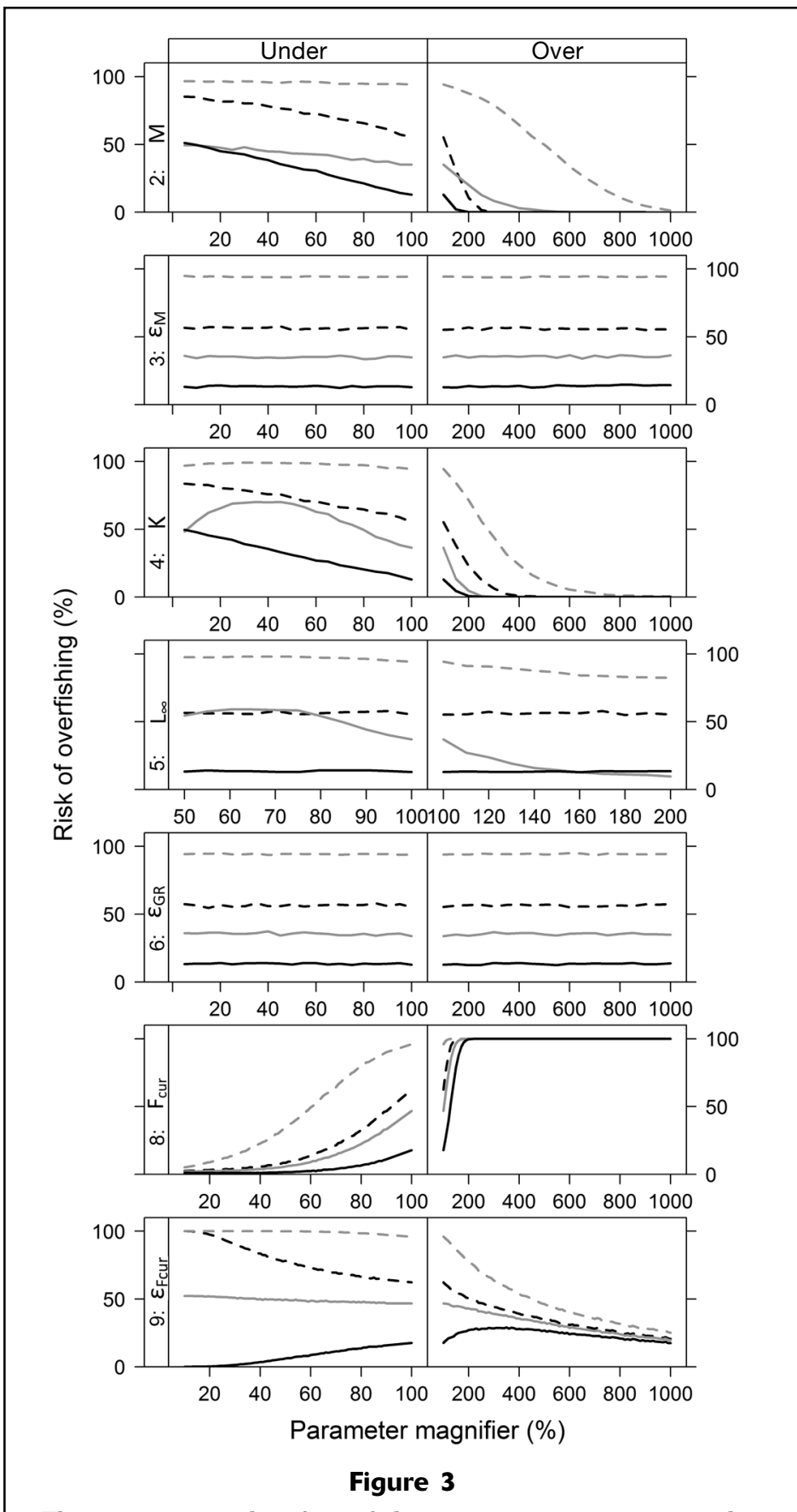

The composite risks of overfishing in scenarios $2-6,8$, and 9 , where the mean and standard deviation (SD) of the natural mortality ( $M$ and $\varepsilon_{\mathrm{M}}$ ), the von Bertalanffy growth coefficient $(K)$, the asymptotic length $\left(L_{\infty}\right)$, the multiplicative error in the growth curve $\left(\varepsilon_{\mathrm{GR}}\right)$, and the mean and $\mathrm{SD}$ of current fishing mortality ( $F_{\text {cur }}$ and $\varepsilon_{\text {Fcur }}$ ) were under-specified from $5 \%$ to $95 \%$ and over-specified from $150 \%$ to $1000 \%$ (except forvalues of $L_{\infty}$ that went from $50 \%$ to $200 \%$ ). The black solid line indicates the composite risk of $F_{\text {cur }}$ exceeding the fishing mortality at which yield per recruit is at its maximum, the black dashed line indicates the composite risk of $F_{\text {cur }}$ exceeding the fishing mortality at which the increase of yield per recruit is only $10 \%$ of the increase of yield per recruit $F=0$, and the gray solid and dashed lines indicate the composite risk of $F_{\text {cur }}$ exceeding the fishing mortality rates at which the spawning biomass per recruit (SPR) is $30 \%$ and $50 \%$ of the SPR when $F=0$. 
The assumption of a gamma distribution of $F_{\text {cur }}$ may be the reason for increasing risks of exceeding $F_{\text {max }}$ when $\varepsilon_{\text {Fcur }}$ lies between $5 \%$ and $200 \%$. When $\varepsilon_{\text {Fcur }}$ was $50 \%$ or less, the gamma distribution of $F_{\text {cur }}$ resembled the normal distribution with density highly concentrated around its mean. Because $F_{\max }(0.151)$ was much larger than $F_{\text {cur }}(0.120)$, the area of the distribution of $F_{\text {cur }}$ that exceeded $F_{\text {max }}$ became smaller and smaller as $\varepsilon_{\text {Fcur }}$ decreased, resulting in lower risks.

\section{Asymmetry in the sensitivities of reference points}

The responses of the means and SDs of $4 F_{\mathrm{BRPs}}$ to the changes in the means of $M$ or $K$ showed disproportionate increases, indicating that the means and SDs of $F_{\text {BRPs }}$ were more sensitive to over-specification. This accelerating trend, arising from the nonlinear relationship between these parameters and $F_{\text {BRPs }}$ (Schnute and Richards, 1998), indicates that under the same degree of misspecification (e.g., 50\%) an over-specified mean of $M$ or $K$ could result in seriously overestimated values of $F_{\text {BRPs }}$. Consequently, the risks of overfishing would be underestimated, potentially leading to overexploitation.

In summary, bias in life history parameters, such as $M, F_{\text {cur }}, K$, and $L_{\infty}$, resulted in considerable changes in the means and SDs of 4 selected $F_{\text {BRPs }}: F_{\max }, F_{0.1}$, $F_{30 \%}$, and $F_{50 \%}$. Different degrees of the imprecision in the life history parameters did not affect the means of $F_{\text {BRPs }}$ but substantially influenced their SDs. Overspecification of the mean of $M$ and $K$ led to larger values of RC in the means and SDs of $F_{\mathrm{BRPs}}$ than did under-specification of the means of $M$ and $K$.

The composite risks of $F_{\text {cur }}$ exceeding these $4 F_{\mathrm{BRPs}}$ were affected mainly by bias in the life history parameters rather than by their imprecision. Both bias and imprecision in $F_{\text {cur }}$ played crucial roles in determining the risks of $F_{\text {cur }}$ exceeding these $4 F_{\text {BRPs. }}$. The variation in growth curves and length-weight relationships did not affect results of the per-recruit models. Our results agreed with those of previous studies without consideration of parameter uncertainty, indicating that they can apply to YPR and SPR models for other species. We recommend 1) minimizing the bias in the life history parameters, especially bias in $M, K$, and $F_{\text {cur }}$, and 2 ) maximizing the precision in $F_{\text {cur }}$ as the most relevant approaches for development of correct estimates of $F_{\mathrm{BRPs}}$ and determining risks of overfishing.

\section{Acknowledgments}

This study is financially supported by the Aims to Top University Project, National Taiwan University. We are thankful to A. Punt, School of Aquatic and Fishery Sciences, University of Washington, for providing comments on the early draft, B. Jessop, Department of Fisheries and Oceans, Bedford Institute of Oceanography, for providing both scientific and grammatical com- ments on the manuscript, and to anonymous reviewers for their suggestions.

\section{Literature cited}

Beverton, R. J. H., and S. J. Holt.

1957. On the dynamics of exploited fish populations, 533 p. Great Britain Fish. Investig. Ser. II. Ministry of Agriculture, Fisheries and Food, Fisheries Laboratory, Lowestoft, U.K.

Chang, Y. J., C.-L. Sun, Y. Chen, S.-Z. Yeh, and W.-C. Chiang. 2009. Incorporating uncertainty into the estimation of biological reference points for a spiny lobster (Panuliruspenicillatus) fishery. N.Z.J. Mar. Freshw. Res. 43:429-442. Article

Charles, A. T.

1998. Living with uncertainty in fisheries: analytical methods, management priorities and the Canadian ground fishery experience. Fish. Res. 37:37-50. Article

Chen, Y., and C. Wilson.

2002. A simulation study to evaluate impacts of uncertainty on the assessment of American lobster fishery in the Gulf of Maine. Can. J. Fish. Aquat. Sci. 59:1394-1403. Article

Cope, J. M., and A. E. Punt.

2007. Admitting ageing error when fitting growth curves: an example using the von Bertalanffy growth function with random effects. Can. J. Fish. Aquat. Sci. 64:205-218. Article

Cubillos, L. A., R. Alarcón, and A. Brante.

1999. Empirical estimates of natural mortality for the Chilean hake (Merlucciusgayi): evaluation of precision. Fish. Res. 42:147-153.

Davey, A. J. H., and D. J. Jellyman.

2003. Sex determination in freshwater eels and management options for manipulation of sex. Rev. Fish Biol. Fish. 15:37-52. Article

Formacion, S. P., J. M. Rongo, and V. C. Sambilay Jr.

1991. Extreme value theory applied to the statistical distribution of the largest lengths of fish. Asian Fish. Sci. 4:123-135. [Available at website.]

Froese, R., and C. Binohlan.

2000. Empirical relationships to estimate asymptotic length, length at first maturity and length at maximum yield per recruit in fishes, with a simple method to evaluate length frequency data. J. Fish Biol. 56:758-773. Article

Gabriel, W. L., and P. M. Mace.

1999. A review of biological reference points in the context of the precautionary approach. In Proceedings of the fifth national NMFS stock assessment workshop: providing scientific advice to implement the precautionary approach under the Magnuson-Stevens Fishery Conservation and Management Act; Key Largo, FL, 24-26 February 1998 (V. R. Restrepo, ed.), p. 34-45. NOAA Tech. Memo. NMFS-F/SPO-40.

Goodyear, C. P.

1993. Spawning stock biomass per recruit in fisheries management: foundation and current use. In Risk evaluation and biological reference points for fisheries management (S. J. Smith, J. J. Hunt, and D. Rivard, eds.), p. 67-81. Can. Spec. Publ. Fish. Aquat. Sci. 120. 
Grabowski, R., and Y. Chen.

2004. Incorporating uncertainty into the estimation of the biological reference points $F_{0.1}$ and $F_{\max }$ for the Maine green sea urchin (Strongylocentrotus droebachiensis) fishery. Fish. Res. 68:367-371. Article

Hall, H. G., S. A. Hesp, and I. C. Potter.

2004. A Bayesian approach for overcoming inconsistencies in mortality estimates using, as an example, data for Acanthopagrus latus. Can. J. Fish. Aquat. Sci. 61:1202-1211. Article

Han, Y.-S., and W.-N. Tzeng.

2006. Use of the sex ratio as a means of resource assessment for the Japanese eel Anguilla japonica: a case study in the Kaoping River, Taiwan. Zool. Stud. 45:255-263.

Helser, T. E., and H.-L. Lai.

2004. A Bayesian hierarchical meta-analysis of fish growth: with an example for North American largemouth bass, Micropterus salmoides. Ecol. Model. 178:339-416. Article

Hilborn, R., and C. J. Walters

1992. Quantitative fisheries stock assessment: choice, dynamics and uncertainty, 570 p. Chapman \& Hall, London.

Jiao, Y., Y. Chen, and J. Wroblewski.

2005. An application of the composite risk assessment method in assessing fisheries stock status. Fish. Res. 72:173-183. Article

Keller, A. A., K. J. Molton, A.C. Hicks, M. Haltuch, and C. Wetzel.

2012. Variation in age and growth of greenstriped rockfish (Sebastes elongatus) along the U.S. west coast (Washington to California). Fish. Res. 119:80-88. Article

King, $\mathrm{M}$.

2007. Fisheries biology, assessment and management, 2nd ed., 400 p. Wiley-Blackwell, Oxford, U.K.

Lai, H. L., and D. R. Gunderson.

1987. Effects of ageing errors on estimates of growth, mortality and yield per recruit for walleye pollock (Theragra chalcogramma). Fish. Res. 5:287-302.

Lin, Y.-J., S.-L. Chang, M.-Y. Chang, S.-H. Lin, T.-I. Chen, M.-

S. Su, W.-C. Su, and W.-N. Tzeng.

$2010 \mathrm{~b}$. Comparison of recapture rates and estimates of fishing and natural mortality rates of Japanese Eel Anguilla japonica between different origins and marking methods in a mark-recapture experiment in the Kaoping River, southern Taiwan. Zool. Stud. 49:616-624.

Lin, Y.-J., Y.-J. Chang, C.-L. Sun, and W. N. Tzeng.

2010a. Evaluation of the Japanese eel fishery in the lower reaches of the Kao-Ping River, southwestern Taiwan using a per-recruit analysis. Fish. Res. 106:329-336. Article

Lin, Y.-J., N.-J. Su, B. M. Jessop, W.-C. Chiang, and C.-L. Sun. 2012. Errors in coefficient or expected value? Effects of different methods on simulated values for a linear model and the nonlinear von Bertalanffy growth model. J. Fish. Soc. Taiwan 39:23-34.

Lin, Y.-J., and C.-L. Sun.

2013. Estimation of natural mortality of Japanese eel (Anguilla japonica) using multiple indirect methods. J. Fish. Soc. Taiwan 40:171-182.
Lin, Y.-J., and W.-N. Tzeng.

2008. Effects of shrimp net and cultured eels on the wild population of Japanese eel Anguilla japonica in the Kao-Ping River, Taiwan. J. Fish. Soc. Taiwan 35:65-73. 2009. Modelling the growth of Japanese eel Anguilla japonica in the lower reach of the Kao-Ping River, southern Taiwan: an information theory approach. J. Fish Biol. 75:100-112. Article

2010. Vital population statistics based on length frequency analysis of the exploited Japanese eel (Anguilla japonica) stock in the Kao-Ping River, southern Taiwan. J. Appl. Ichthyol. 26:424-431. Article

Mace, P. M.

1994. Relationships between common biological reference points used as thresholds and targets of fisheries management strategies. Can. J. Fish. Aquat. Sci. 51:110-122. Article

Pascual, M. A., and O. O. Iribarne.

1993. How good are empirical predictions of natural mortality? Fish. Res. 16:17-24.

Peterman, R. M.

2004. Possible solutions to some challenges facing fisheries scientists and manager. ICES J. Mar. Sci. 61:1331-1343. Article

Prager, M. H., C. E. Porch, K. W. Shertzer, and J. F. Caddy.

2003. Target and limits for management of fisheries: a simple probability-based approach. N. Am. J. Fish. Manage. 23:349-361. Article

Quinn, J. T., II, and R. B. Deriso.

1999. Quantitative fish dynamics, 560 p. Oxford Univ. Press, New York.

$\mathrm{R}$ Core Team.

2014. R: a language and environment for statistical computing. R Foundation for Statistical Computing, Vienna, Austria. [Available at website, accessed May 2014.]

Restrepo, V. R., and W. W. Fox Jr.

1988. Parameter uncertainty and simple yield-per-recruit analysis. Trans. Am. Fish. Soc. 117:282-289. Article

Seber, G. A. F.

1982. The estimation of animal abundance and related parameters, $2^{\text {nd }}$ ed., 672 p. Charles Griffin \& Co., London.

Schnute, J. T., and L. J. Richards.

1998. Analytical models for fishery reference points. Can. J. Fish. Aquat. Sci. 55:515-528. Article

Sissenwine, M. P., and J. G. Shepherd.

1987. An alternative perspective on recruitment overfishing and biological reference points. Can. J. Fish. Aquat. Sci. 44: 913-918. Article

United Nations.

1995. Agreement for the implementation of the provisions of the United Nations Convention on the Law of the Sea of 10 December 1982 relating to the conservation and management of straddling fish stock and highly migratory fish stocks. United Nations Conference on Straddling Fish Stocks and Highly Migratory Fish Stocks, Sixth session, New York, 24 July-4 August 1995. A/CONF. 164/37, 40 p. [Available at website.]

Vetter, E. F.

1988. Estimation of natural mortality in fish stocks: a review. Fish. Bull. 86:25-43. 


\section{Appendix}

This appendix presents changes in composite risks of 2 random variables, $X$ and $Y$, under different means and standard deviations.

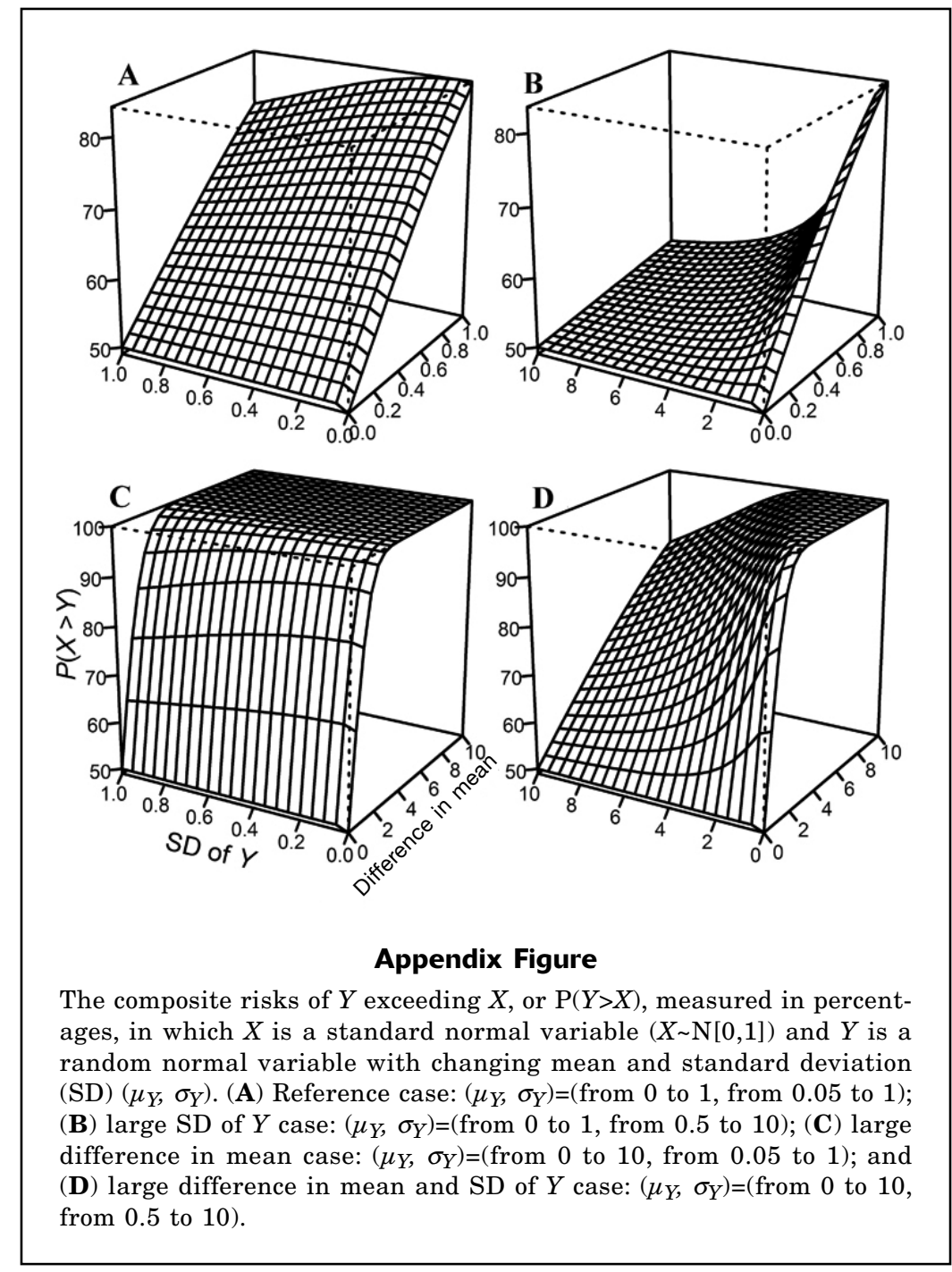

